\title{
MACDONALD POSITIVITY VIA THE HARISH-CHANDRA $D$-MODULE
}

\author{
I.G. GORDON
}

Abstract. Using the Harish-Chandra $D$-module, we give a proof of Haiman's theorem on the positivity of Macdonald polynomials. Ginzburg's work on the connection between this $D$-module and the isospectral commuting variety is fundamental to this approach.

\section{INTRODUCTION}

The (transformed) Macdonald polynomials $\tilde{H}_{\mu}(z ; q, t)$ are symmetric functions with coefficients that are rational functions of two parameters $q$ and $t$. They have remarkable specialisations to important families of symmetric functions including Hall-Littlewood polynomials, Jack polynomials and Schur functions.

Expanding the Macdonald polynomials in terms of Schur functions,

$$
\tilde{H}_{\mu}(z ; q, t)=\sum_{\lambda} \tilde{K}_{\lambda, \mu}(q, t) s_{\lambda}(z)
$$

Macdonald conjectured that the coefficients $\tilde{K}_{\lambda, \mu}(q, t)$ belong to $\mathbb{N}[q, t]$. In a wonderful paper, [7], Haiman confirmed this conjecture by proving the $n$ ! theorem. This showed the existence of a vector bundle $\tilde{\mathcal{P}}$ on Hilb ${ }^{n} \mathbb{C}^{2}$, the Hilbert scheme of points on the plane, with many remarkable properties. In particular, the fibres of $\tilde{\mathcal{P}}$ at the torus fixed points of $\mathrm{Hilb}^{n} \mathbb{C}^{2}$ are bigraded representations of $\mathfrak{S}_{n}$ encoding the Macdonald polynomials. Haiman's proof of the $n$ ! theorem is a remarkable blend of sophisticated algebraic geometry and subtle combinatorics.

In this note we give a different proof of Macdonald positivity using recent work of Ginzburg, [4]. This proof again displays a vector bundle on $\mathrm{Hilb}^{n} \mathbb{C}^{2}$ whose fibres at torus fixed points carry the Macdonald polynomials. The bundle is constructed from a degeneration of the Harish-Chandra $D$-module on the Grothendieck-Springer resolution of type $A_{n-1}$; to describe its fibres requires only standard constructions from $D$-module theory and the Springer correspondence. It should be noted that in [4] Ginzburg showed that this bundle is isomorphic to $\tilde{\mathcal{P}}$ if one assumes Haiman's results. We do not know if it is possible to give a new proof of the $n$ ! theorem along similar lines.

Following Haiman's pioneering work there have been two recent proofs of generalisations of Macdonald positivity, [1] and [5]. These are of a different flavour to this note.

I thank Gwyn Bellamy and Victor Ginzburg for helpful comments. I am grateful for the full financial support of EPSRC grant EP/G007632. 


\section{Positivity}

Let $V$ be an $n$-dimensional complex vector space, $G=G L(V)$ with Lie algebra $\mathfrak{g}=\mathfrak{g l}(V)$, and set $\mathfrak{t}$ to be the subalgebra of $\mathfrak{g}$ consisting of diagonal matrices. Let $B \leq G$ be the Borel subgroup of upper triangular matrices, with Lie algebra $\mathfrak{b}$. The Weyl group, $W=\mathfrak{S}_{n}$, acts on $\mathfrak{t}$. We will identify $\mathfrak{g}$ and $\mathfrak{t}$ with $\mathfrak{g}^{*}$ and $\mathfrak{t}^{*}$ via the trace pairing.

Let $\kappa: \mathfrak{g} \times \mathfrak{g} \rightarrow \mathfrak{g}$ be the commutator. The commuting variety, $\mathfrak{C}$, is the scheme-theoretic fibre $\kappa^{-1}(0)$. Set $\mathfrak{T}=\mathfrak{t} \times \mathfrak{t}$. Simultaneous conjugation provides an action of $G$ on $\mathfrak{C}$ such that the algebraic geometric quotient $\mathfrak{C} / G$ is isomorphic to $\mathfrak{T} / W$, see $[2$, Theorem 1.3]. Let $\mathfrak{X}=$ $[\mathfrak{C} \times \mathfrak{T} / W \mathfrak{T}]_{\text {red }}$, the reduced isospectral commuting variety, and let $\mathfrak{X}_{\text {norm }}$ be its normalisation with morphism $\psi: \mathfrak{X}_{\text {norm }} \longrightarrow \mathfrak{X}$. There is a projection morphism $p_{\mathfrak{C}}: \mathfrak{X} \longrightarrow \mathfrak{C}$ and an induced morphism on the normalisations $p: \mathfrak{X}_{\text {norm }} \longrightarrow \mathfrak{C}_{\text {norm }}$.

There is an action of $G$ on $\mathfrak{X}$ induced from $\mathfrak{C}$, of $\mathbb{C}^{*} \times \mathbb{C}^{*}$ by dilation in both sets of variables, and of $W$ from the diagonal action on $\mathfrak{T}$. All these lift to $\mathfrak{X}_{\text {norm }}$.

Let $\tilde{\mathfrak{g}}=G \times{ }_{B} \mathfrak{b}$ be the Grothendieck-Springer resolution. It admits morphisms $\mu: \tilde{\mathfrak{g}} \rightarrow \mathfrak{g}$ and $\nu: \tilde{\mathfrak{g}} \rightarrow \mathfrak{t}$ defined by $(g, x) \mapsto g x g^{-1}$, respectively $(g, x) \mapsto x \bmod [\mathfrak{b}, \mathfrak{b}]$. Let $\mathcal{M}=\int_{\mu \times \nu} \mathcal{O}_{\tilde{\mathfrak{g}}}$, the

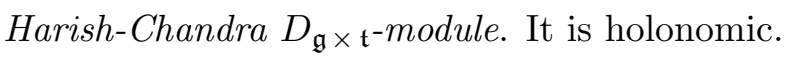

Theorem 1. [4, Theorem 1.3.3, Theorem 1.3.4, Theorem 1.5.2]

(1) There is a filtration on $\mathcal{M}$, the Hodge filtration, such that $\operatorname{gr} \mathcal{M} \cong \psi_{*} \mathcal{O}_{\mathfrak{X}_{\text {norm }}}$.

(2) $\mathfrak{X}_{\text {norm }}$ is Cohen-Macaulay and Gorenstein.

(3) Set $\mathcal{R}=p_{*} \mathcal{O}_{\mathfrak{X}_{\text {norm }}}$. Over the smooth locus of $\mathfrak{C}, \mathcal{R}$ is a $G \times W \times \mathbb{C}^{*} \times \mathbb{C}^{*}$-equivariant vector bundle whose fibres carry the regular representation of $W$.

Let $\mathcal{S}=\{(X, Y, v) \in \mathfrak{g} \times \mathfrak{g} \times V:[X, Y]=0, \mathbb{C}\langle X, Y\rangle v=V\}$. The action of $G$ on $\mathcal{S}$ is free, and its quotient is Hilb $\mathbb{C}^{2}$, the Hilbert scheme of $n$ points on the plane. The $\mathbb{C}^{*} \times \mathbb{C}^{*}$-action on Hilb $\mathbb{C}^{2}$ has a finite number of fixed points, $I_{\mu}$, labelled by partitions of $n$, see for instance [7, §3.2].

The projection morphism from $\mathcal{S}$ to $\mathfrak{g} \times \mathfrak{g}$ has image $\mathfrak{C}^{\circ}$, the set of pairs $(X, Y) \in \mathfrak{C}$ that have a cyclic vector. This makes $\mathcal{S}$ a torsor over $\mathfrak{C}^{\circ}$.

Since $\mathfrak{C}^{\circ}$ is smooth we may define an open set $\mathfrak{X}^{\circ}=p^{-1}\left(\mathfrak{C}^{\circ}\right)$ in $\mathfrak{X}_{\text {norm }}$ and then set $\mathfrak{W}=$ $\left(\mathfrak{X}^{\circ} \times \mathfrak{C}^{\circ} \mathcal{S}\right) / G$. We have the following diagram, see $[4,(8.2 .1)]$



Set $\mathcal{P}=\left(\rho_{*} \delta^{*}\left(\left.\mathcal{R}\right|_{\mathfrak{C}^{\circ}}\right)\right)^{G}$. By [4, Corollary 8.1.3] this is a $W \times \mathbb{C}^{*} \times \mathbb{C}^{*}$-equivariant vector bundle on $\mathrm{Hilb}^{n} \mathbb{C}^{2}$ whose fibres carry the regular representation of $W$. It is shown in $[4, \S 8.2]$ that $\mathfrak{W}$ is isomorphic to the relative spectrum of $\mathcal{P}$, so $\mathcal{P} \cong \eta_{*} \mathcal{O}_{\mathfrak{W}}$. 
The transformed Macdonald polynomials $\tilde{H}_{\mu}(z ; q, t)$ are two parameter symmetric functions attached to partitions $\mu$. They may be characterised by the following conditions in the ring of symmetric functions over the base field $\mathbb{Q}(q, t),[8$, Definition 3.5.2].

(Mi) $\tilde{H}_{\mu}[(1-q) Z ; q, t] \in \mathbb{Q}(q, t)\left\{s_{\lambda}(z): \lambda \geq \mu\right\}$

(Mii) $\tilde{H}_{\mu}[(1-t) Z ; q, t] \in \mathbb{Q}(q, t)\left\{s_{\lambda}(z): \lambda \geq \mu^{t}\right\}$

(Miii) $\tilde{H}_{\mu}[1 ; q, t]=1$.

Here $s_{\lambda}(z)$ is the Schur function attached to the partition $\lambda, \geq$ is the dominance ordering on partiations, and the $[\cdot \cdot]$ denotes plethystic substitution, see $[8, \S 3.3]$.

The following theorem gives another proof of Macdonald positivity. This was proved first by Haiman in [7], and subsequently in [1] and [5]. We do not assert here that $\mathcal{P}$ is the Procesi bundle, although that does follow from the work of Haiman and Ginzburg, see [4, Corollary 8.2.5]. Recall the Frobenius characteristic is the unique linear map from the representation ring of $\mathfrak{S}_{n}$ to symmetric functions, sending the irreducible representation $\lambda$ to the Schur function $s_{\lambda}(z)$, see $[8, \S 3.2]$.

Theorem 2. Let $\mathcal{P}\left(I_{\mu}\right)$ be the fibre of $\mathcal{P}$ above $I_{\mu} \in$ Hilb $^{n} \mathbb{C}^{2}$, which by the above carries a $W \times$ $\mathbb{C}^{*} \times \mathbb{C}^{*}$-action. The Frobenius characteristic $F_{\mathcal{P}\left(I_{\mu}\right)}(z ; q, t)$ equals $\tilde{H}_{\mu}(z ; q, t)$.

The proof of this will occupy the rest of this note. It proceeds in a similar way to the tactic of Haiman's own proof, using however basic facts about $D$-modules.

Any function in $\mathcal{O}(\mathfrak{T})$ pulls back to a regular function on $\mathfrak{X}_{\text {norm }}$, and by construction these functions are invariant under the action of $G$. Thus the functions in $\mathcal{O}(\mathfrak{T})$ give rise to functions on $\mathfrak{W}$ and hence an action on $\mathcal{P}$. Let $y_{1}, \ldots, y_{n}$ be a basis of linear functionals on $\mathfrak{t} \times\{0\} \subset \mathfrak{T}$.

Claim 1. The elements $y_{1}, \ldots, y_{n}$ are a regular sequence at any point in $\mathfrak{W}$ at which they vanish.

Proof. Let $I=\left(y_{1}, \ldots, y_{n}\right)$ be the ideal of $\mathcal{O}_{\mathfrak{W}}$ generated by the $y_{i}$ 's. Thanks to [4, Proposition 3.2.4] $\mathfrak{W}$ is Cohen-Macaulay. Hence it is enough to show that $\operatorname{codim} I=n$. This follows just as in [7, Proposition 3.3.3], for instance.

In $\left[4\right.$, Proposition 3.2.4] it is shown that $\mathfrak{W} \cong\left[\mathrm{Hilb}^{n} \mathbb{C}^{2} \times_{\mathfrak{T} / W} \mathfrak{T}\right]_{\mathrm{red}}$, norm. Since the support of $I_{\mu} \in \mathrm{Hilb}^{n} \mathbb{C}^{2}$ is concentrated at the origin of $\mathfrak{T} / W$, there is a unique point $\left(I_{\mu}, 0\right) \in\left[\operatorname{Hilb}^{n} \mathbb{C}^{2} \times_{\mathfrak{T}} / W\right.$ $\mathfrak{T}]_{\text {red }}$ lying above $I_{\mu} \in$ Hilb $^{n} \mathbb{C}^{2}$ and we let $\mathcal{J}_{\mu}$ be the corresponding maximal ideal sheaf. Let $A=\mathcal{O}_{\left[\text {Hilb }^{n} \mathbb{C}^{2} \times_{\mathfrak{T} / W} \mathfrak{T}\right]_{\text {red }}}$ and $B=\mathcal{O}_{\mathfrak{W}}$. We now know that $\left(y_{1}, \ldots, y_{n}\right)$ is a regular sequence in $\left({ }_{A} B\right)_{\mathcal{J}_{\mu}}$. It follows that $\left({ }_{A} B\right)_{\mathcal{J}_{\mu}} /\left(y_{1}, \ldots, y_{n}\right)\left({ }_{A} B\right)_{\mathcal{J}_{\mu}}$ admits a Koszul resolution, and hence by [8, Proposition 3.3.1] that we have an equality of Frobenius characteristics

$$
F_{\left({ }_{A} B\right)_{\mathcal{J}_{\mu}}}([1-q] Z ; q, t)=F_{\left({ }_{A} B\right)_{\mathcal{J}_{\mu}} /\left(y_{1}, \ldots, y_{n}\right)\left({ }_{A} B\right)_{\mathcal{J}_{\mu}}}(z ; q, t) .
$$

Since $\eta: \mathfrak{W} \longrightarrow$ Hilb $^{n} \mathbb{C}^{2}$ factors through $\left[\operatorname{Hilb}^{n} \mathbb{C}^{2} \times_{\mathfrak{T} / W} \mathfrak{T}\right]_{\mathrm{red}}$, the stalk $\mathcal{P}_{\mu}$ of $\mathcal{P}$ at $I_{\mu}$ equals $\left({ }_{A} B\right)_{\mathcal{J}_{\mu}}$. By freeness $F_{\mathcal{P}_{\mu}}(z ; q, t)=F_{\mathcal{P}\left(I_{\mu}\right)}(z ; q, t) p_{\mu}(q, t)$ where $p_{\mu}(q, t) \in \mathbb{Q}(q, t)$ is the bigraded Poincaré series for the local ring of Hilb $^{n} \mathbb{C}^{2}$ at the point $I_{\mu}$. It follows that

$$
F_{\mathcal{P}\left(I_{\mu}\right)}([1-q] Z ; q, t)=F_{\mathcal{P}_{\mu}}([1-q] Z ; q, t) p_{\mu}(q, t)=F_{\left({ }_{A} B\right)_{\mathcal{J}_{\mu}} /\left(y_{1}, \ldots, y_{n}\right)\left({ }_{A} B\right)_{\mathcal{J}_{\mu}}}(z ; q, t) p_{\mu}(q, t) .
$$


Therefore to check (Mi), we need only show that

$$
F_{\left({ }_{A} B\right)_{\mathcal{J}_{\mu}} /\left(y_{1}, \ldots, y_{n}\right)\left({ }_{A} B\right)_{\mathcal{J}_{\mu}}}(z ; q, t) \in \mathbb{Q}(q, t)\left\{s_{\lambda}(z): \lambda \geq \mu\right\} .
$$

By [6, Proposition 5.3] this is implied by the following.

Claim 2. The $\lambda$ isotypic component of $\left({ }_{A} B\right)_{\mathcal{J}_{\mu}} /\left(y_{1}, \ldots, y_{n}\right)\left({ }_{A} B\right)_{\mathcal{J}_{\mu}}$ is zero unless $\lambda \geq \mu$.

Proof. Since $\mathfrak{C}^{\circ}$ belongs to smooth locus of $\mathfrak{C}$, the restriction of $p: \mathfrak{X}_{\text {norm }} \longrightarrow \mathfrak{C}_{\text {norm }}$ to $\mathfrak{X}^{\circ}$ factors through $\mathfrak{X}$, that is $\left.p\right|_{\mathfrak{X}^{\circ}}=\left.\left(p_{\mathfrak{C}} \circ \psi\right)\right|_{\mathfrak{x}^{\circ}}$. It follows that

$$
\left.\mathcal{R}\right|_{\mathfrak{C}^{\circ}}=p_{*}\left(\mathcal{O}_{\mathfrak{X}_{\text {norm }}} \mid \mathfrak{X}^{\circ}\right)=\left(p_{\mathfrak{C}}\right)_{*}\left(\left.(\operatorname{gr} \mathcal{M})\right|_{p_{\mathfrak{C}}^{-1}\left(\mathfrak{C}^{\circ}\right)}\right) .
$$

Now let $\left(X_{\mu}, Y_{\mu}\right)$ be an element in the principal nilpotent pair orbit corresponding to $\mu$, see $[3$, (0.1)]. We deduce that the stalk of $\mathcal{R}$ above $\left(X_{\mu}, Y_{\mu}\right)$ equals $(\operatorname{gr} \mathcal{M})_{K_{\mu}}$ where $K_{\mu}$ is the maximal ideal of $\left(X_{\mu}, Y_{\mu}, 0,0\right)$, the unique point in $\mathfrak{X}$ lying over $\left(X_{\mu}, Y_{\mu}\right)$.

Let $\pi: \mathfrak{g} \longrightarrow \mathfrak{g} \times \mathfrak{t}$ be the inclusion that sends $X$ to $(X, 0)$. Define

$$
T^{*}(\mathfrak{g})=\mathfrak{g} \times \mathfrak{g}^{*} \stackrel{\rho_{\pi}}{\longleftarrow} \mathfrak{g} \times \mathfrak{g} \times \mathfrak{t} T^{*}(\mathfrak{g} \times \mathfrak{t})=\mathfrak{g} \times \mathfrak{g}^{*} \times \mathfrak{t}^{*} \stackrel{\varpi_{\pi}}{\longrightarrow} T^{*}(\mathfrak{g} \times \mathfrak{t})=\mathfrak{g} \times \mathfrak{g}^{*} \times \mathfrak{t} \times \mathfrak{t}^{*}
$$

by $\rho_{\pi}(X, Y, w)=(X, Y)$ and $\varpi_{\pi}(X, Y, w)=(X, Y, 0, w)$. We set $T_{\mathfrak{g}}^{*}(\mathfrak{g} \times \mathfrak{t})=\rho_{\pi}^{-1}\left(T_{\mathfrak{g}}^{*}(\mathfrak{g})\right)=\mathfrak{g} \times\{0\} \times$ $\mathfrak{t}^{*}$. The characteristic variety of $\mathcal{M}$ is $\operatorname{Ch}(\mathcal{M})=[\mathfrak{X}]$, [4, Corollary 2.4.1]. Now

$$
\begin{aligned}
\varpi_{\pi}^{-1}(\mathfrak{X}) \cap T_{\mathfrak{g}}^{*}(\mathfrak{g} \times \mathfrak{t}) & =\{(X, Y, w):[X, Y]=0, X \text { nilpotent, e-vals }(Y)=w\} \cap\{(X, 0, w)\} \\
& =\{(X, 0,0): X \text { nilpotent }\} \subset \mathfrak{g} \times\{0\} \times\{0\}=\mathfrak{g} \times \mathfrak{g} \times \mathfrak{t} T_{\mathfrak{g} \times \mathfrak{t}}^{*}(\mathfrak{g} \times \mathfrak{t}) .
\end{aligned}
$$

Thus $\pi$ is non-characteristic with respect to $\mathcal{M}$. In particular we deduce from [10, Theorem 4.7] that $\operatorname{Ch}\left(\pi^{*} \mathcal{M}\right)=\rho_{\pi} \omega_{\pi}^{-1}(\operatorname{Ch}(\mathcal{M}))=\{(X, Y):[X, Y]=0, X$ nilpotent $\} \subset \mathfrak{C}$. In fact, the $y_{1}, \ldots, y_{n}$ form a regular sequence for gr $\mathcal{M}$ by [4, Proposition 9.1.3], so multiplication by each $y_{i}$ on $\operatorname{gr} \mathcal{M} /\left(y_{1}, \ldots y_{i-1}\right) \operatorname{gr} \mathcal{M}$ is injective, and iterating the proof of Step 1 of $[10$, Theorem 4.7] shows that $\left(\rho_{\pi}\right)_{*} \varpi_{\pi}^{*}(\operatorname{gr} \mathcal{M})$ is isomorphic to gr $\pi^{*} \mathcal{M}$.

The support of $\left(\rho_{\pi}\right)_{*} \varpi_{\pi}^{*}(\operatorname{gr} \mathcal{M})$ is $\{(X, Y):[X, Y]=0, X$ nilpotent $\}$. Since $\mathcal{M}$ is holonomic this space is lagrangian in $T^{*}(\mathfrak{g})$, a union of conormal bundles $\bigcup_{\lambda} \overline{T_{\mathcal{O}_{\lambda}}^{*}(\mathfrak{g})}$, where $\mathcal{O}_{\lambda}$ denotes the nilpotent orbit in $\mathfrak{g}$ of type $\lambda$. The $D$-module $\mathcal{M}$ carries a $W$-action, $[9, \S 5]$ and this induces the $W$-action that is inherited by $\mathcal{R}$ in the statement of Theorem 1(3). The $\lambda$-isotypic component of the stalk of $\left.\mathcal{R}\right|_{\mathfrak{C}^{\circ}} /\left.\left(y_{1}, \ldots, y_{n}\right) \mathcal{R}\right|_{\mathfrak{C}^{\circ}}$ at $\left(X_{\mu}, Y_{\mu}\right)$ is non-zero if and only if $\left(X_{\mu}, Y_{\mu}\right)$ is in the support of the $\lambda$-isotypic component of $\left(\rho_{\pi}\right)_{*} \varpi_{\pi}^{*}(\operatorname{gr} \mathcal{M})$.

We have a decomposition $\pi^{*} \mathcal{M}=\bigoplus_{\lambda}\left(\pi^{*} \mathcal{M}\right)_{\lambda}$. We've seen above that the support of $\operatorname{gr}\left(\pi^{*} \mathcal{M}\right)_{\lambda}$ equals the support of the $\lambda$-isotypic component of $\left(\rho_{\pi}\right)_{*} \varpi_{\pi}^{*}(\operatorname{gr} \mathcal{M})$. By [9, Proposition 4.8.1 and Theorem 5.3(3)], $\left(\pi^{*} \mathcal{M}\right)_{\lambda}$ is supported on the closure of the nilpotent orbit $\mathcal{O}_{\lambda}$, and so $\operatorname{Ch}\left(\left(\pi^{*} \mathcal{M}\right)_{\lambda}\right) \subseteq \bigcup_{\nu \leq \lambda} \overline{T_{\mathcal{O}_{\nu}}^{*} \mathfrak{g}}$. Thus the $\lambda$-isotypic component of the stalk of $\left.\mathcal{R}\right|_{\mathfrak{C}^{\circ}} /\left.\left(y_{1}, \ldots, y_{n}\right) \mathcal{R}\right|_{\mathfrak{C}^{\circ}}$ at $\left(X_{\mu}, Y_{\mu}\right)$ is non-zero only if $\left(X_{\mu}, Y_{\mu}\right) \in \overline{T_{\mathcal{O}_{\nu}}^{*}(\mathfrak{g})}$ for some $\nu \leq \lambda$. But since $X_{\mu} \in \mathcal{O}_{\mu}$, this in turn requires that $\mu \leq \nu$. So we deduce that the stalk at $\left(X_{\mu}, Y_{\mu}\right)$ of the $\lambda$-isotypic component of $\left.\mathcal{R}\right|_{\mathfrak{C}^{\circ}} /\left.\left(y_{1}, \ldots, y_{n}\right) \mathcal{R}\right|_{\mathfrak{C}^{\circ}}$ is non-zero only if $\mu \leq \lambda$. 
Given any $s \in \mathcal{S}$ we have by definition

$$
\left(\mathcal{P} /\left(y_{1}, \ldots, y_{n}\right) \mathcal{P}\right)_{\rho(s)} \otimes_{\mathcal{O}_{\mathrm{Hib}^{n} \mathbb{C}^{2}, \rho(s)}} \mathcal{O}_{\mathcal{S}, s} \cong\left(\left.\mathcal{R}\right|_{\mathfrak{C}^{\circ}} /\left.\left(y_{1}, \ldots, y_{n}\right) \mathcal{R}\right|_{\mathfrak{C}^{\circ}}\right)_{\delta(s)} \otimes_{\mathcal{O}_{\mathfrak{C}^{\circ}, \delta(s)}} \mathcal{O}_{\mathcal{S}, s}
$$

If $s \in \delta^{-1}\left(X_{\mu}, Y_{\mu}\right)$ then $\rho(s)=I_{\mu}$ and it follows that the $\lambda$-isotypic component of $\mathcal{P}_{\mu} /\left(y_{1}, \ldots, y_{n}\right) \mathcal{P}_{\mu}$ is non-zero only if $\mu \leq \lambda$. Since $\left({ }_{A} B\right)_{\mathcal{J}_{\mu}} /\left(y_{1}, \ldots, y_{n}\right)\left({ }_{A} B\right)_{\mathcal{J}_{\mu}}=\mathcal{P}_{\mu} /\left(y_{1}, \ldots, y_{n}\right) \mathcal{P}_{\mu}$, this proves our claim.

To deal with (Mii) we argue similarly, reducing the calculations about $\mathcal{P}$ to ones on $\mathfrak{X}$. We need to factor out a basis $z_{1}, \ldots, z_{n}$ of $\mathfrak{t}^{*}$. To see this is a regular sequence observe first that there is an automorphism of $\mathfrak{X}$ induced by interchanging $\mathfrak{g} \times \mathfrak{t}$ with $\mathfrak{g}^{*} \times \mathfrak{t}^{*}$. This induces an automorphism of the normalisation $\mathfrak{X}_{\text {norm }}$ and we see that $z_{1}, \ldots, z_{n}$ is a regular sequence since $y_{1}, \ldots, y_{n}$ is. Now recall that $\left(Y_{\mu}, X_{\mu}\right)=\left(X_{\mu^{t}}, Y_{\mu^{t}}\right)$. Thus we deduce that the $\lambda$-isotypic component of $\mathcal{P}_{\mu} /\left(z_{1}, \ldots, z_{n}\right) \mathcal{P}_{\mu}$ is non-zero only if $\mu^{t} \leq \lambda$. This implies (Mii).

Condition (Miii) states that the trivial representation appears in $\mathbb{C}^{*} \times \mathbb{C}^{*}$-bidegree $(0,0)$ and nowhere else. But since $\mathcal{P}_{\mu}$ carries the regular representation of $W$ and the trivial isotypic component is spanned by the constant functions, this is immediate.

\section{REFERENCES}

[1] S. H. Assaf, Dual Equivalence Graphs I: A combinatorial proof of LLT and Macdonald positivity, arXiv:1005.3759.

[2] W.L. Gan and V. Ginzburg, Almost-commuting variety, D-modules, and Cherednik algebras, IMRP 2006:2.

[3] V. Ginzburg, Principal nilpotent pairs in a semisimple Lie algebra 1, Invent. Math. 140, 511-561 (2000).

[4] V. Ginzburg, Isospectral Commuting Variety and the Harish-Chandra D-module, arXiv:1002.3311.

[5] I. Grojnowski and M. Haiman, Affine Hecke algebras and positivity of LLT and Macdonald polynomials, preprint (2007).

[6] M. Haiman, Macdonald polynomials and geometry, in New Perspectives in Geometric Combinatorics, MSRI Publications 37 (1999), 207-254.

[7] M. Haiman, Hilbert schemes, polygraphs, and the Macdonald positivity conjecture, J. Amer. Math. Soc. 14 (2001), 94-1006.

[8] M. Haiman, Combinatorics, symmetric functions and Hilbert schemes, Current Developments in Mathematics 2002, no. 1 (2002), 39-111.

[9] R. Hotta and M. Kashiwara, The invariant holonomic system on a semisimple Lie algebra, Invent. math. 75, 327-358, (1984).

[10] M. Kashiwara, D-modules and Microlocal Calculus, Translations of Mathematical Monographs, A.M.S, Volume 217 (2003).

School of Mathematics and Maxwell Institute for Mathematical Sciences, University of EdinBurgh, James Clerk Maxwell Building, Kings Buildings, Mayfield Road, Edinburgh EH9 3JZ, U.K.

E-mail address: igordon@ed.ac.uk 